\title{
Utilização de Técnicas Multivariadas na Avaliação da Divergência Genética entre Clones de Palma Forrageira (Opuntia ficus-indica Mill.) ${ }^{1}$
}

\section{Carlos Adonai Ferreira², Rinaldo Luiz Caraciolo Ferreira ${ }^{3}$, Djalma Cordeiro dos Santos ${ }^{4}$, Mércia Virginia Ferreira dos Santos ${ }^{3}$, José Antônio Aleixo da Silva ${ }^{3}$, Mário de Andrade Lira ${ }^{5}$, Silmar Gonzaga Molica6}

\begin{abstract}
RESUMO - Avaliou-se, por meio de técnicas multivariadas, a divergência genética entre clones de palma forrageira (Opuntia ficusindica Mill.), em um experimento instalado na Estação Experimental da Empresa Pernambucana de Pesquisa Agropecuária - IPA, Caruaru - PE. O delineamento foi em blocos casualizados, com três repetições. Os tratamentos foram 19 clones de palma do Banco de Germoplasma do IPA. Foram mensuradas: a) medidas em artículos, conforme a ordem: comprimento, largura, espessura, número e peso da matéria verde. b) medidas por planta: presença de espinho, número de artículos por ordem e total, altura total, infestação por cochonilha e peso da matéria verde. Realizaram-se análises de variância univariada (ANOVA) e multivariada (MANOVA), das variáveis canônicas (VC) e de agrupamento (AA). Na ANOVA, foi verificada diferença entre as médias de clones e por meio da MANOVA, diferença entre vetores de médias de clones. Com a aplicação da VC, foi possível reduzir a dimensionalidade original para duas dimensões, com explicação de $85,03 \%$ da variação total. Foi considerada, como característica passível de descarte, a porcentagem de infestação por cochonilha. Na AA discriminaram-se nove grupos. A característica porcentagem de infestação por cochonilha não deve ser incluída no estudo da diversidade genética nas condições estudadas. As características de maior discriminação foram espessuras dos artículos primário, secundário e terciário, número de artículo primário e pesos médios de matéria verde por artículos secundário e terciário. Em um programa de melhoramento de palma forrageira, devem-se considerar o grupo de clones e o desempenho do clone quanto às características de maior relevância agronômica e zootécnica.
\end{abstract}

Palavras-chave: agrupamento, variáveis canônicas

\section{Use of Multivariate Techniques in Genetic Divergence Evaluation among Cactus Forage (Opuntia ficus-indica Mill.) Clones}

\begin{abstract}
Through multivariate techniques the phenetic divergence among clones of cactus forage was evaluated, in an experiment installed at Experimental Station of the Empresa Pernambucana de Pesquisa Agropecuária - IPA, Caruaru - PE. The experimental design was a complete block design, with three blocks. The treatments were 19 clones of cactus forage of the Bank of Germoplasma of IPA. It was measured: a) measures in cladodios, according to the order: length, width, thickness, number and weight of the green matter; b) measures by plant: thorn presence, number of cladodios for order and total, total height, infestation for cochineal and weight of the green matter. It was applied the univariate analyses of variance (ANOVA) and multivariate (MANOVA), the canonical variables (CV) and cluster analysis (CA). In ANOVA difference were verified among the averages of clones. Differences among vectors of averages of clones were detected by means of MANOVA. It was possible to reduce the original dimensionality for two dimensions, that explained of $85.03 \%$ of the total variation, by applicating VC. The infestation percentage by cochineal was considered a characteristics susceptible to discard. In CA was discriminated nine group. In the studied conditions, the characteristic infestation percentage for cochineal should not be included in the study of the genetic diversity; the characteristics of larger discrimination were thickness average for cladodio primary, secondary and tertiary, number of primary cladodio and medium weights of green matter for secondary and tertiary cladodio, in a program of crossing of cactus forage, it must be considered the group of clones and the clone performance as characteristics with higher agronomic relevance and animal science.
\end{abstract}

Key Words: cluster, canonical variables

\footnotetext{
1 Parte da dissertação apresentada pelo primeiro autor ao Programa de Pós-Graduação em Biometria da UFRPE.

2 Licenciado em Matemática, Mestre em Biometria. R. Diógenes Fernandes Távora, 442/204, Casa Caiada, Olinda-PE, CEP: $53130-230$.

E.mail: adonaifer@ig.com.br

3 Professor Adjunto da Universidade Federal Rural de Pernambuco, Rua Manoel de Medeiros, s/n, Dois Irmãos, Recife-PE, CEP: $52171-900$.

Bolsista CNPq. E.mail: rinaldof@ufrpe.br; rmsantos@elogica.com.br; jaaleixo@uol.com.br

4 Pesquisador do IPA. E.mail: djalma@ipa.br

5 Pesquisador do IPA, Bolsista CNPq. E.mail: mlira@ipa.br

6 Professor Adjunto da Universidade Federal Rural do Pernambuco. E.mail: molicasilmar@hotmail.com
} 


\section{Introdução}

A divergência genética entre um grupo de progenitores tem sido avaliada com o objetivo de identificar as combinações híbridas de maior efeito heterótico e maior heterozigose, de tal forma que, em gerações segregantes, se tenha maior possibilidade de recuperação de genótipos superiores (Cruz \& Regazzi, 1994).

A inferência sobre a diversidade genética com base em poucas variáveis tem-se mostrado inadequada, por não abranger a complexidade da interação de influências genéticas e ambientais. É evidente que são úteis à avaliação e a interpretação simultânea de um conjunto relativamente grande de caracteres, a fim de captar a maior quantidade possível de efeitos sofridos. Segundo Cruz \& Regazzi (1994), a utilização de técnicas multivariadas é a alternativa, pois permite combinar as múltiplas informações contidas na unidade experimental, de modo que seja possível executar uma seleção com base num complexo de variáveis, proporcionando ainda, discriminar materiais mais promissores sob vários contextos. Assim, a utilização de técnicas multivariadas poderá identificar combinações híbridas de maior efeito heterótico, aumentando a possibilidade de obter genótipos superiores nas gerações segregantes.

No Nordeste brasileiro e principalmente no Estado de Pernambuco, são cultivadas duas espécies de palma, a Opuntia fícus-indica Mill., com as cultivares Gigante e Redonda, e a Nopalea cochenilifera Salm Dyck, cuja cultivar é a palma Miúda ou Doce. Em trabalhos recentes, o clone IPA-20 da primeira espécie, obtido pelo programa de melhoramento do IPA-UFRPE, vem se destacando como mais produtivo em relação aos demais, com superioridade aproximada de 50\%, quando comparado à palma gigante (Santos et al., 1997).

Os estudos envolvendo a palma forrageira quanto aos aspectos agronômicos (Santos et al., 1998) e zootécnicos, utilizaram análises univariadas e quando muito, análises de regressões múltiplas. Assim, não há registro de estudos quantitativos envolvendo análises multivariadas na avaliação da palma forrageira. Conforme Manly (1994), tais técnicas possuem grande vantagem sobre as técnicas univariadas, pois, nos métodos univariados, há interesse apenas na análise da variação em uma variável aleatória, ao passo que, nas multivariadas, são consideradas diversas variáveis aleatórias ao mesmo tempo, cada uma tendo igual importância no início da análise.
De acordo com Dias et al. (1997), há várias técnicas estatísticas multivariadas que podem ser utilizadas em estudos de divergência genética, tais como Análises de Agrupamento, de Componentes Principais e Discriminante, pois proporcionam enriquecimento das informações extraídas dos dados experimentais. A seleção da análise mais adequada é função da precisão desejada, da facilidade da análise e da maneira como os dados foram obtidos.

O presente trabalho foi realizado objetivando aplicar técnicas multivariadas na avaliação da divergência genética em características morfológicas entre 19 clones de palma forrageira.

\section{Material e Métodos}

\section{Coleta de dados}

$\mathrm{O}$ experimento foi realizado na Estação Experimental de Caruaru, pertencente à Empresa Pernambucana de Pesquisa Agropecuária-IPA, situada a 08o 14'18" de latitude Sul e 35o 55' 20" de longitude oeste e 537m de altitude (Encarnação, 1980).

Os dados apresentados neste trabalho referem-se ao período experimental de janeiro de 1998 a março de 2000.

Foi registrada a precipitação pluvial total de $1026 \mathrm{~mm}$ de janeiro de 1998 a fevereiro de 2000, com temperatura variando entre 16,7 e $33,5^{\circ} \mathrm{C}$.

$\mathrm{O}$ experimento foi instalado no delineamento em blocos casualizados, com três repetições. Os tratamentos foram constituídos de 19 clones de palma forrageira (Opuntia ficus-indica Mill.), oriundos do Banco de Germoplasma da Empresa Pernambucana de Pesquisa Agropecuária (Tabela 1).

O plantio foi realizado em janeiro de 1998, utilizando-se um artículo por cova, no espaçamento de $1,0 \times 0,5 \mathrm{~m}$. A parcela experimental foi composta por uma única fileira contendo sete plantas, sendo úteis cinco plantas, com área útil de $2,5 \mathrm{~m}^{2}$. No plantio, foi realizada adubação orgânica e mineral, conforme análise do solo. Periodicamente, foram realizados tratos culturais, na forma de capina com enxada, em toda a área cultivada.

Em março de 2000, aos dois anos de idade da planta, os artículos foram colhidos e mensurados conforme os seguintes critérios de avaliação:

a) Por artículo conforme a posição na planta: espessura do artículo primário (EAP); espessura do artículo secundário (EAS); espessura do artículo terciário (EAT); comprimento do artículo primário 
Tabela 1 - Clones de palma forrageira (Opuntia ficusindica Mill.) avaliados, Caruaru-PE

Table 1 - Clones of the evaluated cactus forage (Opuntia ficus-indicaMill.), Caruaru-PE

\begin{tabular}{|c|c|c|}
\hline $\begin{array}{l}\text { Clones } \\
\text { Clones } \\
\end{array}$ & $\begin{array}{c}\text { Identificação } \\
\text { Identification }\end{array}$ & $\begin{array}{l}\text { Origem } \\
\text { Origin }\end{array}$ \\
\hline $\mathrm{C} 1$ & Gigante & Pernambuco \\
\hline $\mathrm{C} 2$ & Redonda & Pernambuco \\
\hline C3 & IPA -20 & $*$ \\
\hline $\mathrm{C} 4$ & IPA - $90-18$ & $*$ \\
\hline $\mathrm{C} 5$ & IPA - $90-73$ & $*$ \\
\hline C6 & IPA - $90-75$ & $*$ \\
\hline $\mathrm{C} 7$ & IPA - $90-92$ & $*$ \\
\hline $\mathrm{C} 8$ & IPA - $90-106$ & $*$ \\
\hline C9 & IPA $90-111$ & $*$ \\
\hline $\mathrm{C} 10$ & IPA - $90-115$ & $*$ \\
\hline $\mathrm{C} 11$ & IPA - $90-156$ & $*$ \\
\hline $\mathrm{C} 12$ & 1258 - Additional C. V. & \\
\hline $\mathrm{C} 13$ & 1267 - Alegrian Fodder & \\
\hline $\mathrm{C} 14$ & 1278 - México Fodder & México \\
\hline $\mathrm{C} 15$ & 1294 - México Vegetable & México \\
\hline C16 & 1311 - Marmillon Fodder & \\
\hline $\mathrm{C} 17$ & 1327 - Chile Fruti & Chile \\
\hline $\mathrm{C} 18$ & 1327 - Marmillo Fodder & \\
\hline C19 & Algerian & \\
\hline
\end{tabular}

${ }^{*}$ Clones obtidos pelo Programa de Melhoramento do IPA/UFRPE.

* Clones obtained by the Program of Improvement of IPA/UFRPE.

(CAP); comprimento do artículo secundário (CAS); comprimento do artículo terciário (CAT); largura do artículo primário (LAP); largura do artículo secundário (LAS); largura do artículo terciário (LAT); número de artículos primários (NAP); número de artículos secundários (NAS); número de artículos terciários (NAT); número de artículos quaternários (NAQ); peso da matéria verde por artículo secundário (PMVAS); peso da matéria verde por artículo terciário (PMVAT).

No momento da colheita, os artículos primários foram conservados na planta. Para mensuração do comprimento e largura dos artículos, após a colheita, foi realizado o contorno em papel, objetivando mensurar o maior comprimento e a maior largura, seguindo a metodologia descrita por Santos (1992). A espessura foi mensurada utilizando-se um paquímetro.

b) Por planta: presença de espinho por planta (PEP); número de artículos por planta (NAPL); altura da planta (ATP); porcentagem de infestação por cochonilha (PIC) e peso da matéria verde por planta (PMVP). A avaliação da porcentagem de infestação da planta pelo ataque da cochonilha (Diaspis echinocacti - Bouché, 1833) foi realizada por uma escala de notas, segundo Santos (1992): 1 - Planta não atacada; 2 - Planta com até $25 \%$ de infestação, 3 - 26 a 50\%; 4 - 51 a 75\% e 5 - Acima de $76 \%$.

Foi mensurada a presença de espinhos (PE) utilizando-se uma escala de valores percentuais, variando de 1 a 5: 1 - Ausência de espinhos; 2 - Planta com até $25 \%$ de presença de espinhos; 3 - 26 a 50\%; 4 - 51 a $75 \%$ e 5 - Acima de $76 \%$.

\section{Métodos estatísticos}

\section{Análises de variância univariada e multivariada}

A identificação das diferenças entre clones de palma forrageira foram avaliados pelo modelo $Y_{i j}=\mu+\alpha_{i}+\beta_{j}+e_{i j}$ em que: $Y_{i j}=$ valor observado do $\mathrm{i}$ - ésimo clone no $\mathrm{j}$ - ésimo bloco; $\mu$ - média geral do ensaio; $\alpha_{i}$ - é o efeito do i - ésimo clone, $\mathrm{i}=1,2, \ldots ., 20$;

$\beta_{\mathrm{j}}$ - é o efeito do $\mathrm{j}$ - ésimo bloco, $\mathrm{j}=1,2,3$ e $\mathrm{e}_{\mathrm{ij}}$ - erro aleatório do j - ésima observação no i - ésimo clone.

Para comparação de médias de tratamentos, para as características em que a análise de variância foi significativa, foi utilizado o teste de Scott \& Knott (1974), em nível de 5\% de probabilidade.

Na realização da análise de variância multivariada (MANOVA), adotou-se para a avaliação da diferença entre os vetores de médias dos tratamentos o critério de Wilks, citado por Johnson \& Wichern (1992). Na presença de diferenças significativas entre tratamentos, espera-se sempre obter $\Lambda<1$, e tanto mais significativo quanto menor for o seu valor estimado (Ferreira \& Souza, 1997).

\section{Análise das variáveis canônicas}

Procedeu-se a análise das variáveis canônicas a partir da obtenção da matriz T referente às variâncias e covariâncias dos caracteres $\mathrm{x}_{\mathrm{ij}}$, avaliados nos clones, e a matriz E de variâncias e covariâncias dos resíduos. Esta técnica consistiu na transformação do conjunto de $\mathrm{p}$ caracteres originais em um conjunto que é função linear dos $x_{i}, \sigma$.

Foram verificadas as seguintes propriedades: a) Se Y ij é uma variável canônica, então: $\mathrm{Y}_{\mathrm{ij}}=\mathrm{a}_{1} \mathrm{x}_{\mathrm{i} 1}+\mathrm{a}_{2} \mathrm{x}_{\mathrm{i} 2}+\ldots+\mathrm{a}_{\mathrm{p}} \mathrm{x}_{\mathrm{ip}}$; b) Se $\mathrm{Y}_{\mathrm{ij}}^{\prime}$ é outra variável canônica então: $\mathrm{Y}_{\mathrm{ij}}^{\prime}=\mathrm{b}_{1} \mathrm{x}_{\mathrm{i} 1}+\mathrm{b}_{2} \mathrm{x}_{\mathrm{i} 2}+\ldots+\mathrm{b}_{\mathrm{p}} \mathrm{x}_{\mathrm{ip}} \mathrm{e}$

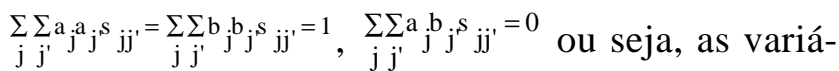
veis canônicas são independentes e $\sigma_{\mathrm{jj}}{ }^{\prime}$ é a covariância residual entre os caracteres $\mathrm{j}$ e $\mathrm{j}^{\prime} \mathrm{e}$ c) A primeira variável canônica $\mathrm{y}_{\mathrm{i} 1}$ apresenta a maior variância, a segunda $\mathrm{y}_{\mathrm{i} 2}$, a segunda maior, e assim sucessivamente. 
$\mathrm{Na}$ análise de variáveis canônicas, o cálculo das variâncias e dos coeficientes de ponderação dos caracteres foi estimado pela resolução do sistema de equação matricial: $\left[T-\lambda_{j} E\right] \mathrm{a}=\Phi$ e a $\mathrm{j}$-ésima variância, pela raiz característica de ordem correspondente, obtida pela solução de: $\operatorname{det}\left[\mathrm{T}-\lambda_{\mathrm{j}} \mathrm{E}\right]=0 \mathrm{em}$ que: a - autovetor associado a cada estimativa das raízes características (coeficientes de ponderação); e $\lambda_{j}$ - matriz representante dos autovalores (raízes características).

A estimação das variáveis canônicas foi obtida a partir de dados transformados estabelecidos por um processo de condensação pivotal, conforme Cruz \& Regazzi (1994), onde os novos caracteres zi's apresentam matrizes de covariâncias residuais nulas e variâncias iguais a um.

Quando definido o número de variáveis canônicas, que absorveram o mínimo de $80 \%$ da variação total, os escores destas foram estimados para cada tratamento, possibilitando a representação gráfica da divergência genética entre os clones, bem como a sua quantificação por meio da matriz de Distância de Maholanobis.

A avaliação do grau de distorção nas distâncias gráficas entre os clones, provocado pela redução de dimensão, foi estimada por $(1-\alpha)$, em que $\alpha$ é a porcentagem acumulada da variância explicada pelas n primeiras variáveis canônicas.

O estudo de descarte de variáveis baseou-se no princípio de que a importância relativa das variáveis canônicas decresce da primeira para a última, pois as últimas variáveis são responsáveis pela explicação de uma fração mínima da variância total disponível (Cruz, 1990). Assim, o caráter que apresentou maior coeficiente de ponderação, em módulo, nas últimas variáveis canônicas, foi considerado de menor importância para explicar a variabilidade em estudo, portanto, passível de descarte.

Vale ressaltar que, se uma variável canônica possuía menor variância e maior coeficiente de ponderação, associado a um caráter já previamente descartado, optou-se por não fazer nenhum outro descarte com base nos coeficientes daquela variável canônica, mas prosseguir a identificação da importância relativa dos caracteres na outra variável imediatamente superior.

A cada identificação de variável passível de descarte foi realizada reanálise do conjunto de remanescente. $\mathrm{O}$ processo de descarte se repetiu até o limite de definição, quando nenhuma distorção na análise de agrupamento pelo método de Tocher foi admitida.

\section{Análise de agrupamento}

A análise de agrupamento foi aplicada utilizando-se como medida de dissimilaridade, a distância de Mahalanobis com base nos dados padronizados remanescente ao descarte de caracteres, por intermédio da técnica de variáveis canônicas.

Para delimitação dos grupos, foram utilizados os métodos de otimização de Tocher, conforme descritos por Souza et al. (1997).

As análises estatísticas foram processadas por meio do SAS 8.0 e do MINITAB 12.0.

\section{Resultados e Discussão}

Os resultados da análise de variância univariada (ANOVA), referentes a 20 caracteres avaliados em 19 clones de palma forrageira, encontram-se sumarizados na Tabela 2. Pode-se observar a existência de diferença significativa $(\mathrm{P}<0,01)$ entre as médias dos tratamentos para maioria dos caracteres avaliados, com exceção da porcentagem por infestação por cochonilha (PIC), observando-se diferença não significativa pelo teste $\mathrm{F}(\mathrm{P}>0,05)$.

As estimativas dos coeficientes de variação foram relativamente baixas para as características referentes à espessura (EAP, EAS e EAT), ao comprimento (CAP, CAS e CAT) e à largura dos artículos (LAP, LAS e LAT), bem como para presença de espinhos na planta (PEP), com valores inferiores a $10 \%$. Para número de artículos de quarta ordem (NAQ), foi observado valor considerado alto, no entanto, vale ressaltar que o número de artículos de quarta ordem ainda não estava definido, devendo o mesmo aumentar com o avanço da idade da planta.

A ocorrência de diferenças significativas entre os clones de certa forma já era esperada, tendo em vista que se trata de materiais de palma forrageira que possuem características fenotípicas contrastantes. Esta situação, segundo Cruz\& Regazzi (1994), éuma indicação bastante favorável ao estudo da divergência genética.

Para as características, em que a análise de variância foi significativa (Tabela 2), foram identificadas diferenças $(\mathrm{P}<0,05)$, por meio do teste de Scott e Knott, sendo observado a formação de dois a cinco grupos de médias (Tabela 3 ). 
Tabela 2 - Resumo da análise da variância univariada (ANOVA) para caracteres de palma forrageira, Caruaru - PE Table 2 - Summary of the univariate analysis of variance (ANOVA) for cactus forage caracters, Caruaru - PE

\begin{tabular}{|c|c|c|c|c|c|}
\hline \multirow[t]{2}{*}{$\begin{array}{l}\text { Caracteres } \\
\text { Characters }\end{array}$} & \multicolumn{3}{|c|}{$\begin{array}{l}\text { Quadrado médio } \\
\text { Mean square }\end{array}$} & \multirow[t]{2}{*}{$\begin{array}{l}\text { Média } \\
\text { Mean }\end{array}$} & \multirow[t]{2}{*}{$\begin{array}{c}\text { Coeficiente de variação }(\%) \\
\text { Coefficient of variation }(\%)\end{array}$} \\
\hline & $\begin{array}{c}\text { Blocos } \\
\text { Blocks }\end{array}$ & $\begin{array}{l}\text { Clones } \\
\text { Clones }\end{array}$ & $\begin{array}{c}\text { Resíduo } \\
\text { Error }\end{array}$ & & \\
\hline EAP & 0,9357 & $0,7253 * *$ & 0,1011 & 3,52 & 9,0 \\
\hline EAS & 0,2802 & $0,5925^{* *}$ & 0,0459 & 2,44 & 8,8 \\
\hline EAT & 0,0216 & $0,4378 * *$ & 0,0286 & 1,85 & 9,1 \\
\hline CAP & 14,0439 & $19,7073 * *$ & 4,4813 & 27,28 & 7,8 \\
\hline CAS & 22,0419 & $21,9365 * *$ & 1,6045 & 32,05 & 4,0 \\
\hline CAT & 10,6684 & $25,0156 * *$ & 2,8393 & 34,31 & 4,9 \\
\hline LAP & 0,8587 & $18,1506 * *$ & 1,3819 & 16,65 & 7,1 \\
\hline LAS & 6,3079 & $22,6924 * *$ & 0,8137 & 17,59 & 5,1 \\
\hline LAT & 0,0919 & $20,4087 * *$ & 0,7403 & 18,79 & 4,6 \\
\hline NAP & 0,3871 & $2,9792 * *$ & 0,2002 & 3,48 & 12,9 \\
\hline NAS & 1,5254 & $10,4982 * *$ & 1,5418 & 8,38 & 14,8 \\
\hline NAT & 12,1342 & $20,3653 * *$ & 2,6233 & 7,03 & 23,0 \\
\hline NAQ & 8,0842 & $5,7123 * *$ & 1,8939 & 2,02 & 68,2 \\
\hline PMVAS & 0,0841 & $0,3183^{* *}$ & 0,0557 & 0,93 & 25,3 \\
\hline PMVAT & 0,0295 & $0,0453 * *$ & 0,0089 & 0,75 & 12,5 \\
\hline PEP & 0,1978 & $0,1208 * *$ & 0,0352 & 2,72 & 6,9 \\
\hline NAPL & 23,6593 & $68,2309 * *$ & 7,6086 & 18,89 & 14,6 \\
\hline ATP & 123,5480 & $509,5361 * *$ & 118,0827 & 98,73 & 11,0 \\
\hline PIC & 0,2416 & $0,4029^{\text {n.s }}$ & 0,2224 & 2,63 & 17,9 \\
\hline PMVP & 71,3091 & $65,6875 * *$ & 12,5138 & 14,10 & 25,1 \\
\hline GL & 2 & 18 & 35 & - & - \\
\hline
\end{tabular}

Por meio da análise de variância multivariada (MANOVA), foram evidenciadas também diferenças significativas $(\mathrm{P}<0,01)$ entre os vetores de médias dos clones (Tabela 4). Dessa forma, com a rejeição da hipótese de que os vetores de médias de clones são iguais, justifica-se o uso de outras técnicas multivariadas, objetivando a redução de dimensão e, ou, descarte de variáveis.

A redução da dimensionalidade proporcionada pelas técnicas de variáveis canônicas encontra-se ilustrada na Tabela 5. Pode-se observar que houve redução para duas dimensões e as duas primeiras variáveis canônicas foram responsáveis pela explicação de $85,03 \%$ da variação total.

Com base nos resultados, justifica-se a utilização da análise de variáveis canônicas, por proporcionarem uma simplificação estrutural dos dados originais, de modo que a divergência, a princípio, influenciada por um conjuntop-dimensional, possa ser representada no espaço bidimensional, de fácil interpretação geométrica.

A concentração da variação nas primeiras combinações lineares de técnicas multivariadas, possivelmente, deve-se ao fato de a maioria dos caracteres envolvidos no estudo, de âmbito morfológico, possuir natureza agronômica, representando, em alguma extensão, os componentes de produção.

A divergência genética foi expressa visualmente através de gráfico, utilizando os escores das duas primeiras variáveis canônicas (Figura 1). Observa-se que os clones C2 e C19 são os mais divergentes.

Com base nos valores contidos na Tabela 6, constatou-se que as características de menor importância, em ordem de descarte, com maior coeficiente de ponderação nas últimas variáveis canônicas, foram peso da matéria verde por artículo secundário (PMVAS) e terciário (PMVAT), presença de espinho por planta (PEP), espessura dos artículos primário (EAP), secundário (EAS) e terciário (EAT), largura do artículo terciário (LAT), número de artículos primários (NAP) e porcentagem de infestação por cochonilha (PIC). No entanto, apenas PIC, poderia ser descartada por não apresentar importância nos coeficientes de ponderação nas duas primeiras variáveis canônicas e nenhuma distorção no processo de agrupamento pelo método de Tocher.

Em razão da própria variância dos dados apresentados na análise univariada (Tabela 3) e dos altos coeficientes de ponderação nas duas primeiras variáveis 


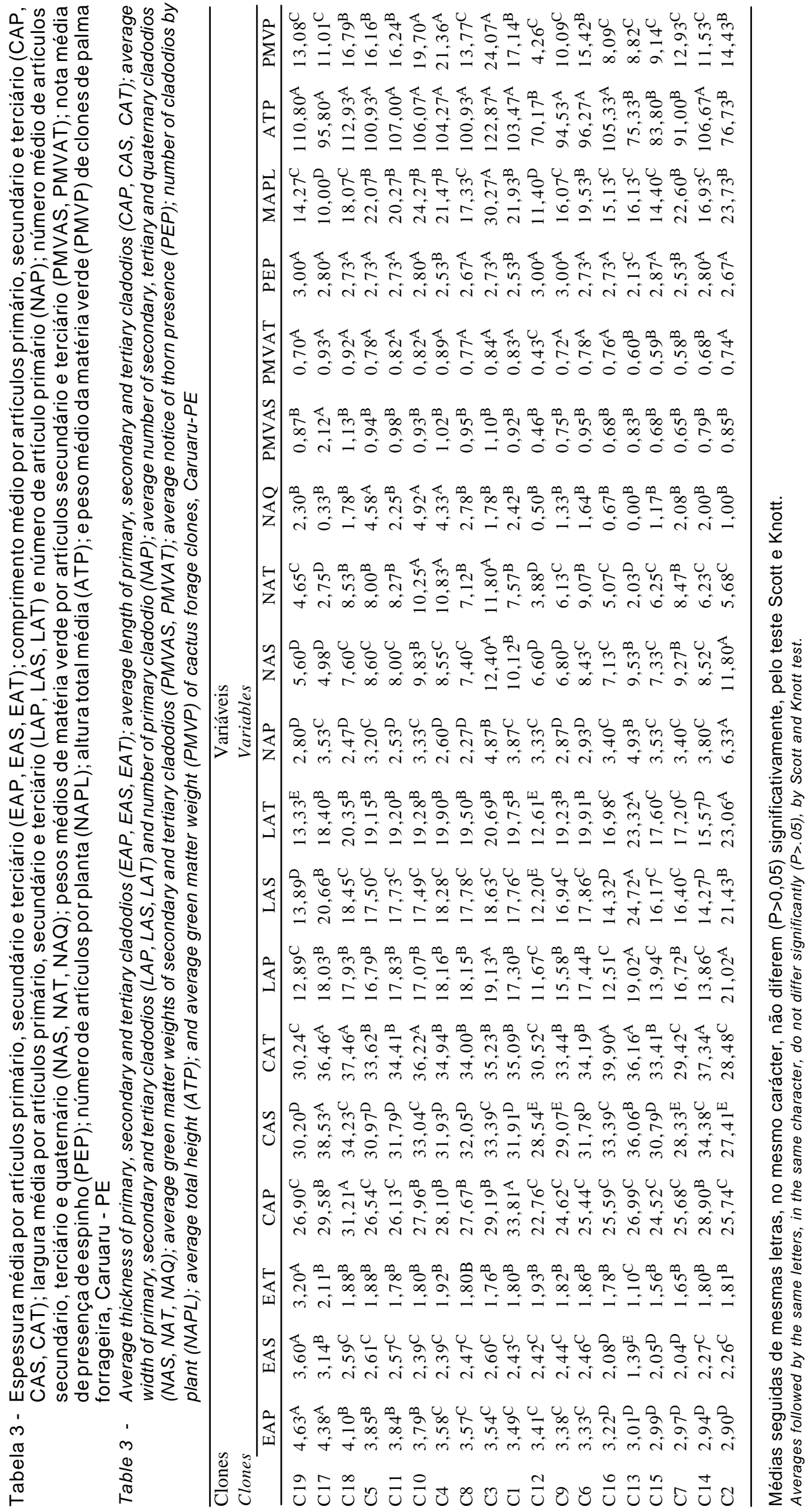


Tabela 4 - Análise de variância multivariada (MANOVA) para clones de palma forrageira, Caruaru - PE

Table 4 - Multivariate analysis of variance (MANOVA) for cactus forage clones, Caruaru-PE

\begin{tabular}{|c|c|c|c|c|c|}
\hline & & & $\begin{array}{l}\text { Teste de W } \\
\text { Wilks lam }\end{array}$ & & \\
\hline $\begin{array}{l}\text { Fonte de variação } \\
\text { Source of variation } \\
\end{array}$ & $\Lambda$ & $\mathrm{F}$ & $\begin{array}{l}\text { GL num } \\
\text { num } D F\end{array}$ & $\begin{array}{l}\text { GL den } \\
\text { den } D F\end{array}$ & $\operatorname{Pr}>\mathrm{F}$ \\
\hline $\begin{array}{l}\text { Clones } \\
\text { Clones }\end{array}$ & 0,00000000 & 4,24 & 360 & 270,76 & $<0,0001$ \\
\hline $\begin{array}{l}\text { Blocos } \\
\text { Blocks }\end{array}$ & 0,04090281 & 2,66 & 40 & 32 & 0,0006 \\
\hline
\end{tabular}

Tabela 5 - Estimativas das variâncias (autovalores, $\lambda_{j} \geq 1$ ) associados às variáveis canônicas (VC) e suas importâncias relativas e acumuladas, obtidas de vinte caracteres avaliados em clones de palma forrageira, Caruaru - PE

Table 5 - Estimates of the variances (eigenvalues, $\lambda_{j} \geq 1$ ) associated to the canonical variables (CV) and their relative and accumulated importances, obtained with twenty characters in cactus forage clones, Caruaru-PE

\begin{tabular}{|c|c|c|c|}
\hline & \multicolumn{3}{|c|}{$\begin{array}{c}\text { Variáveis canônicas } \\
\text { Canonical variates }\end{array}$} \\
\hline & $\begin{array}{c}\text { Variância (Autovalores } \lambda_{\mathrm{j}} \text { ) } \\
\text { Variance (Eigenvalues } \lambda_{j} \text { ) }\end{array}$ & $\begin{array}{c}\text { Variância }(\%) \\
\text { Variance }(\%)\end{array}$ & $\begin{array}{c}\text { Variância acumulada (\%) } \\
\text { Accumulated variance }\end{array}$ \\
\hline $\mathrm{VC} 1(C V 1)$ & 230,2305 & 74,34 & 74,34 \\
\hline $\mathrm{VC} 2(C V 2)$ & 33,1146 & 10,69 & 85,03 \\
\hline $\mathrm{VC} 3(C V 3)$ & 18,1806 & 5,87 & 90,90 \\
\hline $\mathrm{VC} 4(C V 4)$ & 7,8111 & 2,52 & 93,43 \\
\hline VC5 (CV5) & 7,2655 & 2,35 & 95,77 \\
\hline VC6 (CV6) & 4,2324 & 1,37 & 97,14 \\
\hline $\mathrm{VC} 7(C V 7)$ & 2,8804 & 0,93 & 98,07 \\
\hline $\mathrm{VC} 8(C V 8)$ & 1,7615 & 0,57 & 98,64 \\
\hline $\mathrm{VC} 9(C V 9)$ & 1,4394 & 0,46 & 99,10 \\
\hline
\end{tabular}

canônicas, foram consideradas discriminantes as características compreendidas por espessuras dos artículos primário (EAP), secundário (EAS) e terciário (EAT), número de artículo primário (NAP) e pesos médios de matéria verde por artículos secundário e terciário (PMVAS, PMVAT).

Apesar da inspeção visual de gráfico de dispersão, por intermédio dos escores em eixos cartesianos, representados pelas duas primeiras variáveis canônicas, apresentar baixa distorção $(14,97 \%)$ em relação à matriz de distância de Mahalanobis, foi conveniente a utilização da análise de agrupamento para a formação de grupos, estabelecendo grupos com grau de homogeneidade intra-grupo e heterogeneidade extragrupo, baseada na matriz de dissimilaridade.

Dessa forma, os resultados da utilização da análise de agrupamento estimada pelo método de otimização de Tocher, aplicado ao conjunto dos caracteres estudados, sem a característica PIC, possi-

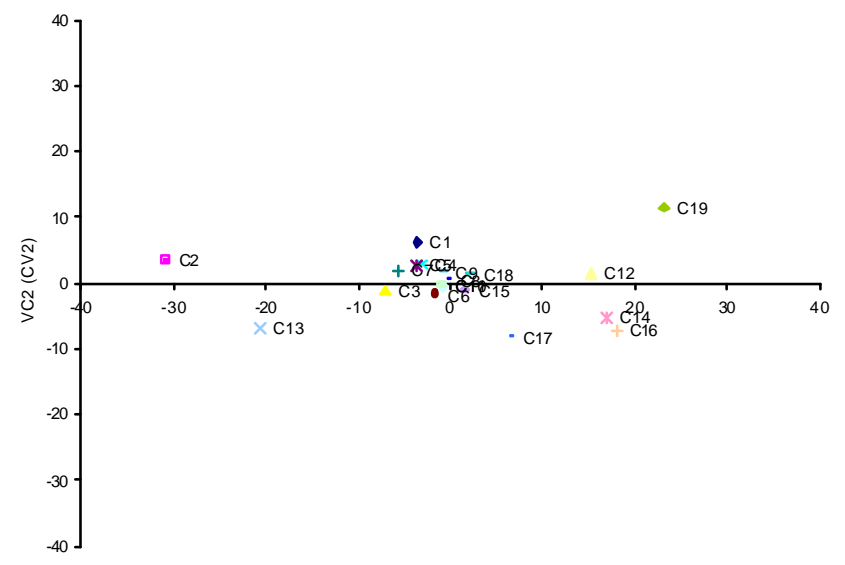

VC1 (CV1)

Figura 1 - Dispersão de escores de clones de palma forrageira em relação às duas primeiras variáveis canônicas (VC1 e VC2).

Figure 1 - Dispersion of the clones scores of cactus forage in relation to the first two canonical variables (VC1 and VC2). 
Tabela 6 - Estimativas dos coeficientes de ponderação associados às últimas variáveis canônicas, Caruaru-PE Table 6 - Estimates of the weighed coefficients associated to the last canonical variables, Caruaru-PE

\begin{tabular}{|c|c|c|c|c|c|c|c|c|c|}
\hline \multirow{3}{*}{$\begin{array}{l}\text { Carácter } \\
\text { Character }\end{array}$} & \multicolumn{8}{|c|}{$\begin{array}{c}\text { Últimas variáveis canônicas } \\
\text { Last canonical variables }\end{array}$} & \multirow[b]{2}{*}{ VC10 } \\
\hline & VC18 & VC17 & VC16 & VC15 & VC14 & VC13 & VC12 & VC11 & \\
\hline & $V C 18$ & VC17 & VC16 & $V C 15$ & $V C 14$ & $V C 13$ & $V C 12$ & $V C 11$ & VC10 \\
\hline EAP & $-0,7657$ & 0,1689 & 0,6668 & $-1,9552$ & - & - & - & - & - \\
\hline EAS & $-1,2117$ & 2,1444 & $-0,5634$ & 1,0431 & $-1,3702$ & $-1,5238$ & - & - & - \\
\hline EAT & 0,2746 & $-2,2894$ & $-1,4328$ & $-0,8221$ & $-2,2614$ & - & - & - & - \\
\hline CAP & 0,0326 & $-0,0879$ & $-0,0380$ & $-0,0035$ & $-0,0750$ & 0,0271 & $-0,0755$ & $-0,0126$ & $-0,0520$ \\
\hline CAS & 0,3789 & 0,2734 & 0,2037 & $-0,5685$ & 0,0693 & $-0,4094$ & $-0,3083$ & $-0,2270$ & 0,3810 \\
\hline CAT & $-0,4318$ & $-0,2648$ & $-0,3770$ & 0,3827 & $-0,1787$ & 0,3451 & 0,0272 & 0,3451 & $-0,2068$ \\
\hline LAP & $-0,3549$ & $-0,1496$ & $-0,1238$ & $-0,1142$ & 0,3046 & $-0,4838$ & 0,3429 & $-0,2219$ & 0,0556 \\
\hline LAS & 0,0738 & 0,0672 & $-0,8119$ & 0,8917 & 0,1408 & 0,3929 & 0,2699 & $-0,2179$ & $-0,4913$ \\
\hline LAT & 0,0368 & $-0,0739$ & 0,8218 & $-0,7319$ & $-0,4183$ & $-0,0254$ & $-0,8957$ & 0,4851 & 0,5182 \\
\hline NAP & $-0,7630$ & 0,4003 & 0,8795 & $-0,6235$ & 1,0580 & $-0,2109$ & 0,6336 & $-0,8210$ & 0,6887 \\
\hline NAS & 0,1516 & 0,0392 & $-0,5974$ & 0,1675 & 0,0429 & $-0,2348$ & 0,0447 & $-0,0643$ & $-0,2405$ \\
\hline NAT & $-0,3081$ & 0,2123 & $-0,1192$ & $-0,4055$ & 0,3093 & 0,1622 & 0,0492 & $-0,4556$ & 0,1740 \\
\hline NAQ & $-0,0967$ & 0,0659 & 0,1347 & $-0,0153$ & 0,3062 & $-0,1978$ & $-0,0707$ & 0,3435 & 0,5359 \\
\hline PMVAS & $-1,0403$ & $-2,5926$ & 1,0387 & 0,2816 & $-1,7373$ & 4,5888 & $-1,4921$ & 4,3027 & $-0,2994$ \\
\hline PMVAT & 7,9198 & 8,2161 & $-4,5423$ & $-0,3985$ & 4,6860 & $-7,2807$ & 11,5856 & $-4,5020$ & 0,2237 \\
\hline PEP & 1,8958 & $-1,3423$ & $-2,3671$ & 1,3870 & 2,4372 & $-0,0784$ & $-0,6153$ & $-0,4666$ & $-1,1865$ \\
\hline NAPL & 0,1462 & $-0,0711$ & $-0,0910$ & $-0,0326$ & $-0,2480$ & 0,2396 & $-0,1106$ & 0,4969 & $-0,2843$ \\
\hline ATP & $-0,0081$ & $-0,0013$ & 0,0569 & 0,0297 & 0,0410 & $-0,0331$ & 0,0246 & $-0,0315$ & $-0,0194$ \\
\hline PIC & $-0,4718$ & 0,5035 & $-0,2006$ & $-0,4450$ & 1,3317 & 0,3928 & $-0,5469$ & 0,3317 & $-0,5571$ \\
\hline PMVP & 0,0291 & $-0,0972$ & 0,1617 & 0,2559 & $-0,0780$ & 0,0630 & $-0,0545$ & $-0,2017$ & 0,1177 \\
\hline
\end{tabular}

- Refere-se ao descarte do caráter associado ao coeficiente correspondente.

- Refers to the discard of the character associated to the corresponding coefficient.

bilitaram discriminar nove grupos distintos (Tabela 7). Assim, como sugestão para um programa de melhoramento com o gênero Opuntia de palma forrageira, deve-se considerar o grupo de clones, em vez dos clones individualmente. Nesse sentido, a decisão do melhorista será selecionar clones parentais, com base nas distâncias intergrupos (Tabela 8), cruzando grupos mais distantes. Vale salientar que não basta considerar a divergência entre agrupamentos como único critério para orientar os cruzamentos. O desempenho do clone deve ser considerado, sobretudo quando são envolvidos as características de maior relevância agronômica e zootécnica.

Os resultados da análise de agrupamento atesta-
Tabela 7 - Grupos de dissimilaridade entre clones de palma forrageira estabelecidos pelo método de Tocher a partir das Distâncias de Mahalanobis, Caruaru - PE

Table 7 - Dissimilarity groups among cactus forage clones established for the method of Tocher starting from the Mahalanobis distance, Caruaru-PE

\begin{tabular}{lccc}
\hline $\begin{array}{l}\text { Grupos } \\
\text { Groups }\end{array}$ & & $\begin{array}{c}\text { Clones } \\
\text { Clones }\end{array}$ & \\
\hline I & C1 & C2 & \\
II & C3 & C4 & C19 \\
III & C5 & C6 & \\
IV & C7 & C8 & \\
V & C9 & C10 & \\
VI & C11 & C12 & \\
VII & C13 & C14 & \\
VIII & C15 & C16 & \\
IX & C17 & C18 & \\
\hline
\end{tabular}


Tabela 8 - Estimativas de distâncias de Mahalanobis $\left(D^{2}\right)$ intra e intergrupo referentes a 19 caracteres avaliados em clones de palma forrageira, Caruaru-PE

Table 8 - Estimates of Mahalanobis distance (D2) intra and intergroup regarding 19 evaluated characters in cactus forage clones, Caruaru-PE

\begin{tabular}{|c|c|c|c|c|c|c|c|c|c|}
\hline Grupos & I & II & III & IV & $\mathrm{V}$ & VI & VII & VIII & IX \\
\hline $\bar{I}$ & 753,97 & 361,11 & 903,86 & 412,71 & 477,09 & 865,06 & 893,34 & 1072,63 & 754,88 \\
\hline II & & 30,87 & 316,48 & 19,36 & 26,64 & 220,53 & 416,75 & 326,43 & 143,30 \\
\hline III & & & 533,60 & 274,92 & 237,53 & 257,56 & 694,67 & 335,31 & 266,31 \\
\hline IV & & & & 30,19 & 13,77 & 175,69 & 410,75 & 272,62 & 110,87 \\
\hline V & & & & & 4,93 & 129,73 & 397,81 & 210,63 & 72,69 \\
\hline VI & & & & & & 261,90 & 541,97 & 169,40 & 116,47 \\
\hline VII & & & & & & & 1406,00 & 567,58 & 424,49 \\
\hline VIII & & & & & & & & 302,31 & 131,21 \\
\hline IX & & & & & & & & & 106,99 \\
\hline
\end{tabular}

I - C1 e C2; II - C3 e C4; III - C5, C6 e C19; IV - C7 e C8; V - C9 e C10; VI - C11 e C12; VII - C13 e C14; VIII - C15 e C16; IX - C17 e C18. $I-C 1$ and $C 2 ; I I-C 3$ and $C 4 ; I I I-C 5, C 6$ and $C 19 ; I V-C 7$ and $C 8 ; V-C 9$ and $C 10 ; V I-C 11$ and $C 12 ; V I I-C 13$ and $C 14 ; V I I I-C 15$ and $C 16 ; I X-C 17$ and $C 18$.

rama consistência da metodologia de descarte utilizada, quanto à pouca importância da característica eliminada (PIC), em relação ao estudo da divergência, pois a formação dos grupos (Tabela 7) foi semelhante à obtida a partir do conjunto dos 20 caracteres originais, o que reforça a pouca importância da característica descartada.

\section{Conclusões}

A característica porcentagem de infestação por cochonilha não deve ser incluída no estudo da diversidade genética nas condições estudadas.

As características de maior discriminação foram espessuras dos artículos primário secundário e terciário, número de artículo primário e pesos médios de matéria verde por artículos secundário e terciário.

Em um programa de melhoramento com o gênero Opuntia de palma forrageira, devem-se considerar o grupo de clones e o desempenho do clone quanto às características de maiorrelevânciaagronômicaezootécnica.

\section{Literatura Citada}

CRUZ, C.D. Aplicação de algumas técnicas multivaridas no melhoramento de plantas. Piracicaba: Escola Superior de Agricultura "Luiz de Queiroz", 1990. 188p. Tese (Doutorado em Melhoramento Genético) - Escola Superior de Agricultura "Luiz de Queiroz", 1990.

CRUZ, C.D. ; REGAZZI, A.J. Divergência genética. In: CRUZ, C.D.; REGAZZI, A.J. (Eds.) Métodos biométricos aplicados ao melhoramento genético. Viçosa, MG: Universidade Federal de Viçosa, 1994. p.287-323.

DIAS, L.A.S.; KAGEYAMA, P.Y.; CASTRO, G.C.T. et al. Divergência fenética multivariada na preservação de germoplasma de cacau (Theobroma cacao L.). Revista Agrotrópica, v.9, n.1, p.29-40, 1997.
ENCARNAÇÃO, C.R.F. Observações metereológicas e tipos climáticos das unidades experimentais da empresa IPA. Recife: IPA, 1980. p.110.

FERREIRA, R.L.C.; SOUZA, A.L. Técnicas de análise multivariada aplicadas ao manejo florestal. Viçosa, MG: Universidade Federal de Viçosa, 1997. 21p. (Boletim Técnico SIF, 14).

JOHNSON, R.A.; WICHERN, D.W. Applied multivariate statistical analysis. 2.ed. Englewood Cliffs: Prentice-Hall, 1992. 642p.

MANLY, F.J. Multivariate statistical methods: a primer. London: Chapman and Hall, 1994. 215p.

SANTOS, D.C. Estimativa de parâmetros e caracteres de clones de palma forrageira (Opuntia fícus indica Mill e Nopalea cochenillifera Salm Dyck). Recife:Universidade Federal Rural de Pernambuco, 1992. 119p. Dissertação (Mestrado em Produção Animal) - Universidade Federal Rural de Pernambuco, 1992.

SANTOS, D.C.; FARIAS, I.; LIRA, M.A. et al. A palma forrageira (Opuntia ficus indica Mill e Nopalea cochenillifera Salm Dyck) em PE: cultivo e utilização. Recife: IPA, 1997. 23p. (Documentos, 25)

SANTOS, D.C.; LIRA, M.A.; SANTOS, M.V.F. et al. Competição de clones de palma forrageira (Opuntia e Nopalea), São Bento do Una-PE. In: REUNIÃO ANUAL DA SOCIEDADE BRASILEIRA DE ZOOTECNIA, 35., 1998, Botucatu. Anais... Botucatu: Sociedade Brasileira de Zootecnia, 1998. p.37-39.

SCOTT, A.J.; KNOTT, M. A cluster analysis method for grouping means in the analysis of variance. Biometrics, v.30, n.3, p.507-512, 1974.

SOUZA, A.L.; FERREIRA, R.L.C.; XAVIER, A. Análise de agrupamento aplicada à ciência florestal. Viçosa, MG: Universidade Federal de Viçosa, 1997. 109p. (Documento SIF, 16) 